\title{
Biodegradable Core-Shell Carriers for Simultaneous Encapsulation of Synergistic Actives
}

Maike Windbergs, ${ }^{*}, \dagger, \S, \|$ Yuanjin Zhao, ${ }^{\dagger, \perp}$ John Heyman, ${ }^{\dagger, \#}$ and David A. Weitz ${ }^{*, \dagger, \nabla}$

${ }^{\dagger}$ School of Engineering and Applied Sciences and ${ }^{\nabla}$ Department of Physics and Kavli Institute for Bionano Science and Technology, Harvard University, Cambridge, Massachusetts 02138, United States

${ }^{\text {tD}}$ Department of Biopharmaceutics and Pharmaceutical Technology, Saarland University, Campus A 4.1, 66119 Saarbruecken, Germany

${ }^{\S}$ Helmholtz Institute for Pharmaceutical Sciences Saarland, Saarbruecken, Germany

"Centre for BioMicrosystems, Korea Institute of Science and Technology, Seoul, Republic of Korea

${ }^{\perp}$ State Key Laboratory of Bioelectronics, Southeast University, 210096 Nanjing, China

\#Habsel Inc., Cambridge, Massachusetts 02142, United States

Supporting Information

ABSTRACT: Simultaneous encapsulation of multiple active substances in a single carrier is essential for therapeutic applications of synergistic combinations of drugs. However, traditional carrier systems often lack efficient encapsulation and release of incorporated substances, particularly when combinations of drugs must be released in concentrations of a prescribed ratio. We present a novel biodegradable core-shell carrier system fabricated in a one-step, solvent-free process on a microfluidic chip; a hydrophilic active (doxorubicin hydrochloride) is encapsulated in the aqueous core, while a hydrophobic active (paclitaxel) is encapsulated in the solid shell. Particle size and composition can be precisely controlled, and core and shell can be individually loaded with very high efficiency. Drugloaded particles can be dried and stored as a powder. We demonstrate the efficacy of this system through the simultaneous encapsulation and controlled release of two synergistic anticancer drugs using two cancerderived cell lines. This solvent-free platform technology is also of high potential value for encapsulation of other active ingredients and chemical reagents.

\section{INTRODUCTION}

Efficient delivery of drugs or other active ingredients often entails a specific vehicle for encapsulation and release. Such a vehicle must effectively protect the active from environmental conditions, while providing a suitable means for efficient release of the drug. As drug therapies become increasingly sophisticated, the synergistic benefits of two or more drugs are often required; this is particularly true for conditions, such as cancer or HIV, where drug cocktails are often used. ${ }^{1,2}$ Combining drugs into single carriers represents a challenge for encapsulation and release technologies. This can be accomplished using powdered forms of drugs by mixing two distinct powders and compressing the mixture into tablet or by creating a carrier structure containing one drug and then coating it with a mixture containing the second drug. ${ }^{3,4}$ However, these methods do not ensure simultaneous delivery of both drugs to the same location at a controlled relevant concentration and is even more difficult, when the drugs have different physicochemical characteristics, such as hydrophilic and hydrophobic actives. 5 To address this issue, polymersomes composed of amphiphilic synthetic block copolymers have been created to include a hydrophilic active in the aqueous core and a hydrophobic active in the shell; both actives are released upon a switch in $\mathrm{pH} .{ }^{6}$ In a second system, a self-assembling polymer nanostructure platform has been used to create nanoparticles containing chemotherapeutics with different solubility profiles. ${ }^{7,8}$ Although these carrier structures provide simultaneous delivery of both actives at the same location at a controlled relative concentration, neither of the systems can be considered generic; the block copolymers for polymersomes must be compatible with the drugs to be encapsulated, and in case of nanoparticles, a drug-polymer with self-assembling properties must first be synthesized. In both cases, the polymers must be biocompatible. These characteristics limit the general applicability of the methods, and some actives will be incompatible with either technique. Other typical fabrication techniques of such structures do not provide an efficient means of encapsulating high-value actives, and/or they entail the use of

Received: February 7, 2013

Published: April 30, 2013 

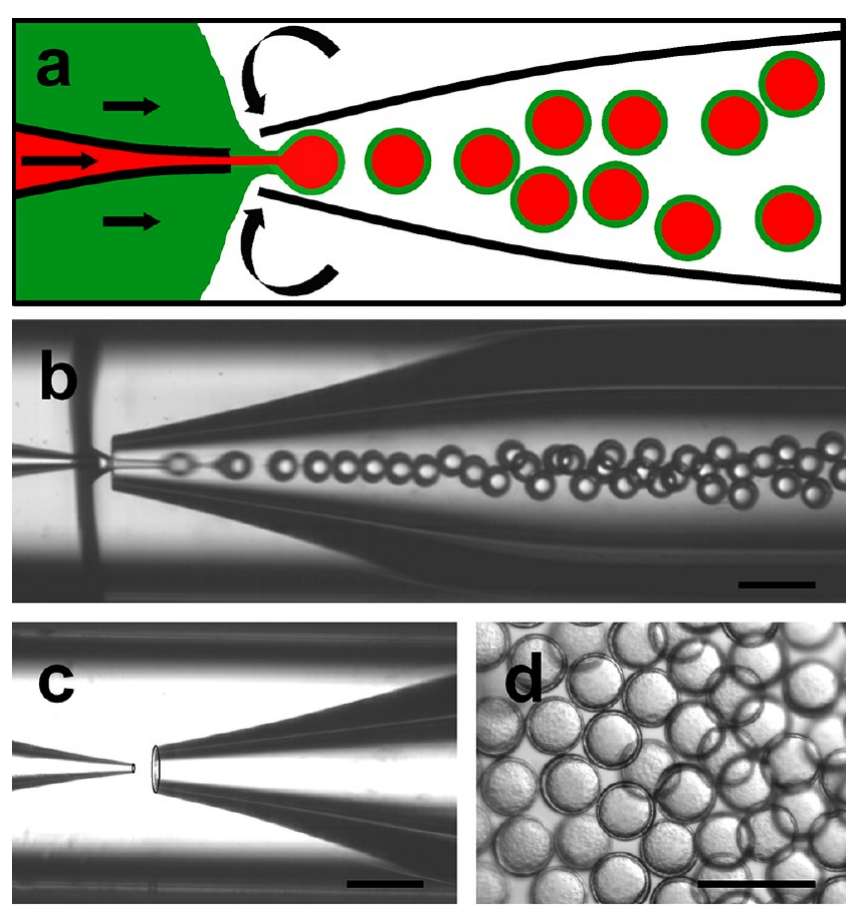

Figure 1. Generation of double emulsion droplets for the formation of core-shell structures in a microcapillary device. (a) Schematic of the device with two adjacently adjusted cylindrical glass capillaries within a square capillary. The device allows the controlled injection of three different fluids. The inner (red) and middle (green) fluids are hydrodynamically focused by the outer fluid (white). (b) Microscopic image of a running device producing core-shell structures. (c) Tapered orifices of the capillaries inside the microfluidic device with a diameter of 20 and $100 \mu \mathrm{m}$, respectively (orifices are highlighted by black circles as a guide to the eye). (d) Microscopy image of coreshell particles as the final product. The scale bars denote $200 \mu \mathrm{m}$. nonbiocompatible solvents which can be harmful even in small, residual quantities. ${ }^{9-11}$

Thus, although powerful delivery systems have been developed for some drug combinations, generic and highly efficient encapsulation and release platform techniques are required to fully exploit synergistic drug administration.

In this paper, we present a generic, solvent-free one-step platform technology to simultaneously encapsulate both, a hydrophilic and a hydrophobic drug with high efficiency, ensuring the controlled release of each active at the same location in the desired range of concentration. We fabricate a core-shell structure consisting of a core of an aqueous solution of the hydrophilic active and a shell of a biodegradable, solid, food-grade lipid in which the hydrophobic active is encapsulated. By using a microfluidic device, we achieve high encapsulation efficiency and precise control of particle size and composition. ${ }^{12-15}$ Particles can be stored as dry powder. We demonstrate the effectiveness of these structures by combining two anticancer drugs which are known to work synergistically and demonstrating their effectiveness using cell viability tests. This solvent-free platform technology is also of high potential for encapsulation of other substances.

\section{RESULTS AND DISCUSSION}

As a vehicle to simultaneously encapsulate two drugs, one hydrophilic and one hydrophobic, we use a double emulsion, in which three separate fluids form the final system. ${ }^{16-18}$ The intermediate fluid can be solidified or gelled and provides an additional barrier separating the innermost and the outermost fluid. Thus, core-shell particles can be produced with improved stability by comparison to an emulsion. ${ }^{19-23}$

As the intermediate fluid, we use a food-grade lipid which is biodegradable and classified as generally recognized as safe (GRAS) substance by the Food and Drug Administration. ${ }^{24-26}$ The capillary microfluidic device consists of two tapered cylindrical
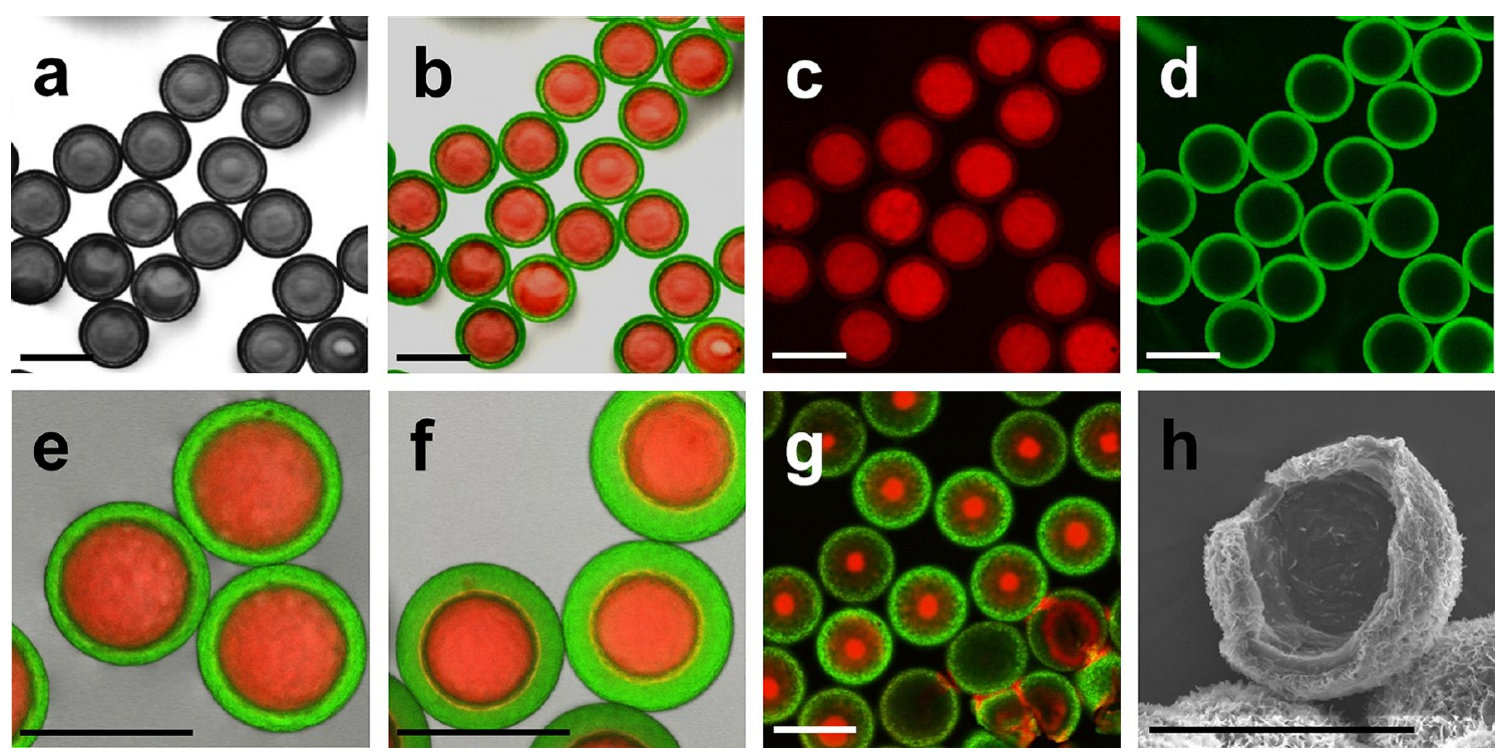

Figure 2. Core-shell particles with the shell consisting of a resolidified lipid and the core formed by an aqueous solution. (a) Light microscopy image depicting the particles in aqueous suspension. (b) Fluorescence detection of the encapsulated drugs: doxorubicin (red) in the core liquid and paclitaxel (green) incorporated within the lipid shell strucure. Overlay image. Single component detection for (c) doxorubicin and (d) paclitaxel. The single component images demonstrate that core and shell phases do not show any mixing phase or overlapping signals. (e,f) Different core-shell mass ratios allowing individual encapsulation of drugs within core and shell phase. $(\mathrm{g})$ Solidified particles. The shell structure remains intact upon solidification, and both actives remain effectively encapsulated upon drying. The lower right corner of the figure depicts particles which we crushed on purpose to release the drug for comparison to confirm that the hydrophilic drug remains encapsulated. (h) Electron microscopy image of a cracked lipid shell. The scale bars denote $100 \mu \mathrm{m}$. 

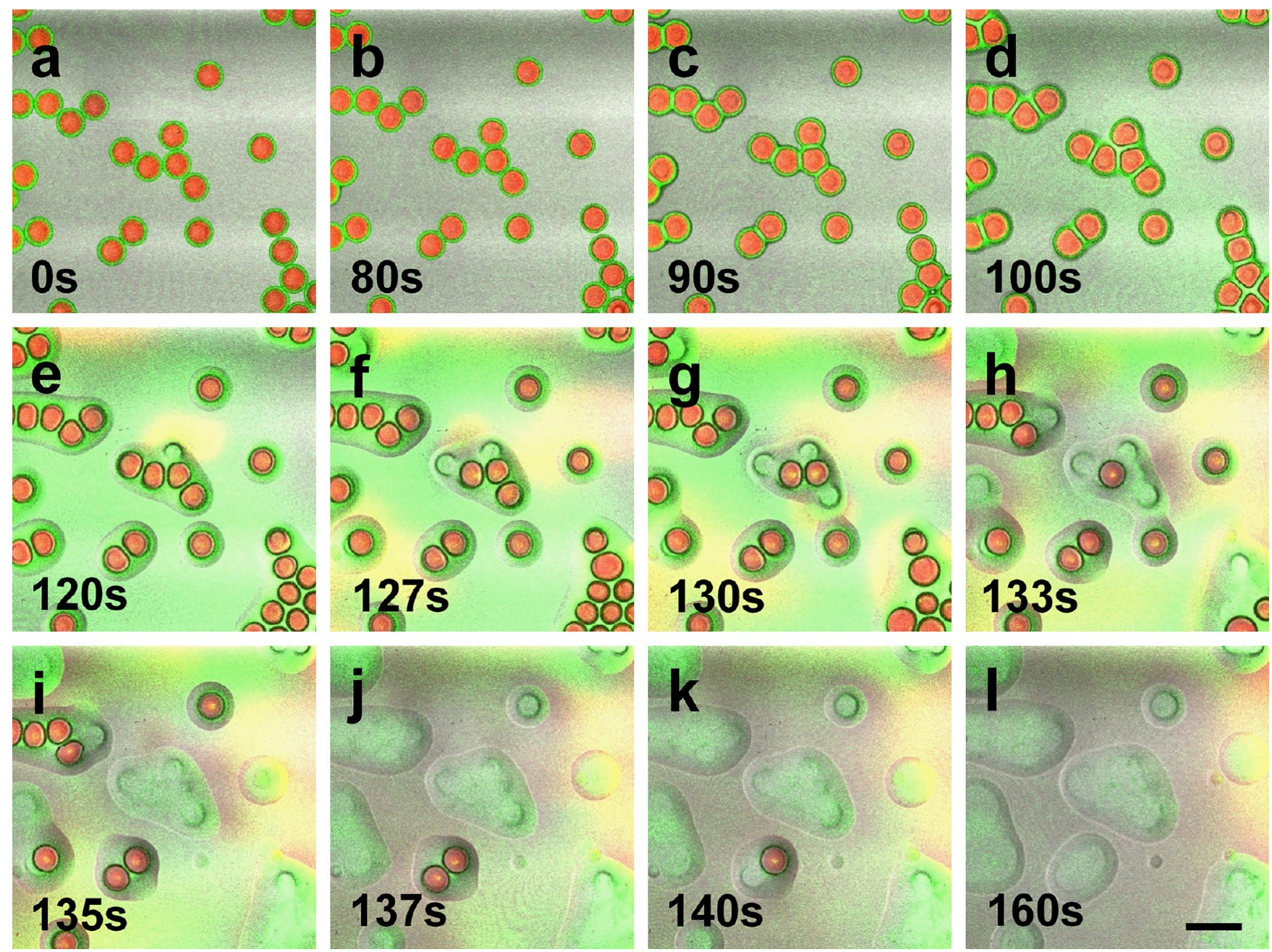

Figure 3. Controlled release of the two encapsulated drugs visualized with fluorescence spectroscopy. To demonstrate simultaneous release of doxorubicin and paclitaxel, we perform in vitro release studies at $37^{\circ} \mathrm{C}$. The particle shell is composed of a lipid with a melting range near body temperature (33.5-35.5 ${ }^{\circ} \mathrm{C}$ ), thus the actives are released upon melting of the shell. The release can be detected by the temporal evolution of the fluorescence of doxorubicin in red and paclitaxel in green color. A video of the process is provided as Supporting Information (video V2). The scale bar denotes $200 \mu \mathrm{m}$.

capillary tubes nested within a square capillary whose inner dimensions match the outer diameters of the cylindrical capillaries, ensuring simple, yet effective alignment of the two tapered orifices, as shown in Figure $1 a-c^{27}$ The inner aqueous phase is injected through the cylindrical capillary, shown on the left in Figure 1a; the orifice has a diameter of $20 \mu \mathrm{m}$. The lipid which forms the shell is injected, in a molten state, from the left through the interstices of the square capillary surrounding the leftmost cylindrical capillary in Figure 1a-c. The outermost fluid is water containing 10 wt \% polyvinylalcohol (PVA) and is injected from the right through the interstices surrounding the right cylindrical capillary, shown in Figure 1c. This fluid flow focuses the coaxial flow of two other fluids, and as a result, drops, with a core-shell structure, are formed by the dripping mechanism at the orifice of the collection capillary, which has a diameter of $100 \mu \mathrm{m}$, as shown in Figure $1 .^{27-29}$ The flow is controlled using syringe pumps allowing the flow rates of each fluid to be adjusted; this controls both, droplet size as well as the relative thickness of the shell (video V1, Supporting Information). To validate our basic concept, we use two common anticancer drugs which are known to act synergistically as representative active ingredients. The first is doxorubicin hydrochloride, which is hydrophilic and is dissolved in the innermost fluid at a concentration of $2 \mathrm{mg} / \mathrm{mL}$. The second is paclitaxel, which is hydrophobic and is dissolved in the lipid at a concentration of $67 \mu \mathrm{g} / \mathrm{mL}$. To enable visualization, we use paclitaxel labeled with the dye Oregon Green, whereas doxorubicin is intrinsically fluorescent. This allows the use of fluorescent microscopy to monitor the behavior of each drug. When they are first produced, the drops are multiple emulsions, consisting of an innermost aqueous core surrounded by a uniform of molten lipid. The liquid state of the shell when the drops are first formed is reflected by its transparent nature, as shown in Figure 1d. The drops are collected in a reservoir immersed in ice water to accelerate solidification of the lipid. This causes the shell to become opaque, as shown in Figure 2a. The drops are highly monodisperse in size, as can be seen in Figures $1 \mathrm{~d}$ and 2.

The two drugs are maintained in separate, yet adjacent compartments in the same particle as visualized by the composite fluorescent image, where doxorubicin is indicated by red and paclitaxel is indicated by green, shown in Figure $2 b$. The individual fluorescent images of doxorubicin (red) and paclitaxel (green) are shown in Figure $2 c, d$, respectively. The concentration of each drug can be individually adjusted; moreover the relative concentrations can also be adjusted by controlling the thickness of the shell as shown in Figure 2e,f. 
Importantly, both drugs are simultaneously encapsulated at the desired concentrations, in the same individual particles, thus ensuring delivery of both actives at the same location for optimum synergistic activity. Moreover, the use of capillary microfluidics to form these particles in a one-step process obviates the need for filling the particles with the drugs post production and ensures very high encapsulation efficiency, as no excess material is generated.

The particles can be filtered and subsequently dried after they are solidified, ensuring long-term stability of the particles and robust encapsulation of the drugs. Both actives remain effectively encapsulated upon drying as shown by the fluorescent images of intact shells in the upper left portion of Figure $2 \mathrm{~g}$. To confirm that the hydrophilic drug remains encapsulated, we crush several particles to release the drug and compare images of these particles in the lower right corner of Figure $2 \mathrm{~g}$. The solid structure of the shell is illustrated by the electron micrograph of a shell that has been crushed and broken, shown in Figure $2 \mathrm{~h}$. These figures confirm the uniform thickness of the shell which helps ensure the encapsulation effectiveness. Furthermore, filtering and drying allow the particles to be packed as a free-flowing powder. Thus, therapeutic application of the particles can be performed either via a suspension which is directly injected into tissue or via the oral route preferentially as dried particles packed within a gelatin capsule for individual drug dosing. Furthermore, because no solvents are used in their synthesis, the particles are ready for use immediately upon fabrication.

The drugs are released by melting the lipid shell. We choose a lipid with a melting range near body temperature $\left(33.5-35.5^{\circ} \mathrm{C}\right)$; however, the structure and hence the melting temperature of the lipid can be chosen to match the specific therapeutic application. Particles based on lipids with a higher melting range would consequently not melt in the human body but rather release their drug load via diffusion and biodegradation. In the gastrointestinal tract, lipids are prone to enzymatic digestion by endogenous lipases, thus facilitating drug release in the intestine. Combinations of individual particles based on low- and high-melting lipids can even be used to provide a mixture of immediate and prolonged drug release, enabling the option for initial and maintenance drug dosing in one application.

To demonstrate simultaneous release of the two drugs, we perform in vitro studies at $37{ }^{\circ} \mathrm{C}$ and detect the temporal evolution of the fluorescence of each. As the lipid shell melts, the hydrophobic drug embedded within it is released, as shown in Figure 3 (video V2, Supporting Information).

Moreover, as the shell becomes fluidized, the inner phase droplets can move freely within the molten lipid and ultimately coalesce with the surrounding phase, thereby releasing the hydrophilic drug. The lipid used in these experiments was chosen to have a rapid release profile upon heating to body temperature.

Alternatively, a lipid with a melting temperature above that of the body will result in a slower release. In this case, release can be achieved by enzymatic degradation rather than melting. In particular, enzymatic release may be useful for depot systems, such as implants, because in human tissue, lipids caused less perturbation than polymer materials. ${ }^{30}$ Due to the broad variety of available lipids, our system offers a platform for many different kinds of therapeutic applications.

To confirm the efficacy of the encapsulated drugs, we conduct viability studies with two different cell lines: ED 19, a
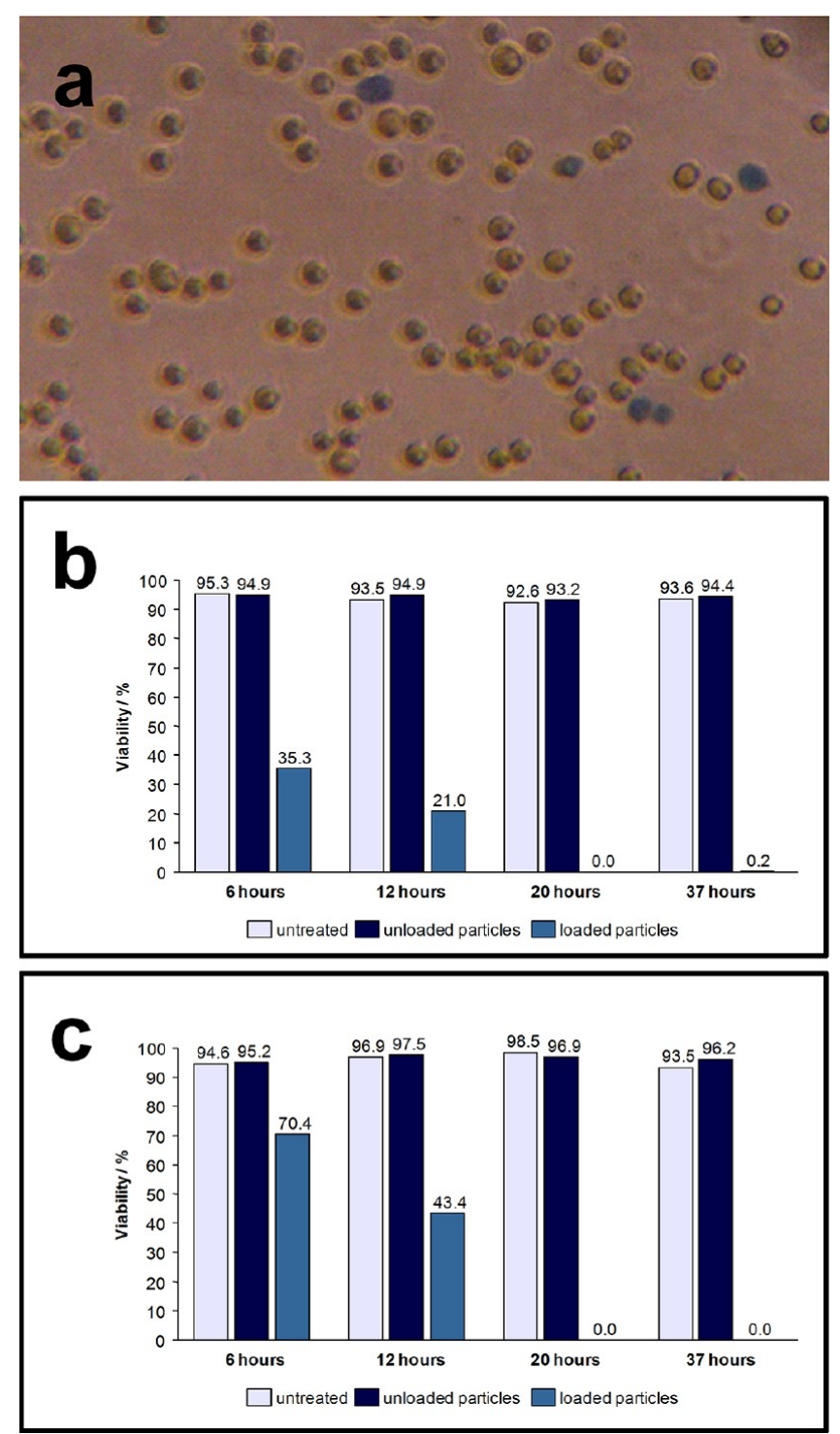

Figure 4. Cell viability studies. (a) Detection of dead cells with a livedead assy based on trypan blue staining. Trypan blue can only transverse cell membranes of dead cells. The microscopy image visualizes stained dead cells (blue) among bright living cells. (b) Viability studies with mouse hybridoma cells (ED 19) and (c) cancerous human cervical cells (HeLa S3). Both cell lines were treated with unloaded and drug-loaded particles, untreated cells served as a control. Unloaded particles did not affect the cell viability, whereas drug-loaded particles clearly cause cell death, thus demonstrating the effectiveness of the released drugs.

mouse hybridoma cell line, and HeLa S3, an immortal cell line derived from cancerous human cervical cells. ${ }^{31,32}$ To determine the effectiveness of the drug treatment, we use a live-dead assay based on trypan blue, which can traverse the cell membrane only when cells are dead. Hence, dead cells are colored blue after staining and can easily be detected among bright living cells, as depicted in Figure 4a.

Both cell lines are incubated with $100 \mu \mathrm{m}$ diameter particles loaded with the drugs, and the fraction of dead cells is determined as a function of time. As control experiments, cells are incubated in the absence of any particles and with particles containing no drugs. As expected due to the GRAS status of the lipid itself, the unloaded core-shell particles do not cause any 
cell death. In contrast, treatment with drug-loaded particles clearly results in cell death. After $6 \mathrm{~h}$ of incubation, ED and HeLa cells show viability rates of $35.3 \%$ and $70.4 \%$, respectively.

After $12 \mathrm{~h}$ the viability is reduced to $21 \%$ for ED cells and $43.4 \%$ for HeLa cells, as different cell types have differing susceptibility to the drug. After $20 \mathrm{~h}$ the viability rates are $0 \%$ for both cell lines (Figure $4 \mathrm{~b}, \mathrm{c}$ ). This demonstrates the effectiveness of the delivered drugs.

\section{CONCLUSIONS}

Our novel biodegradable core-shell carrier system provides a promising approach to encapsulate two substances totally separated from each other. The release profile can be varied through the use of different lipids. The concentrations of the two drugs can be individually adjusted both through their concentrations and through the relative thickness of the shell. Several features of the system are of particular importance for drug delivery applications: Particle formation is precisely controlled, and highly efficient encapsulation for two substances can individually be achieved within a one-step process. Particles can be dried and redispersed while retaining their efficacy. The carrier material is biodegradable, and the process is solvent-free. Thus, this biodegradable system represents a promising new platform technology for the simultaneous, highly efficient, solvent-free encapsulation and favorable release of multiple ingredients.

\section{ASSOCIATED CONTENT}

\section{S Supporting Information}

Experimental details and two videos, one showing the generation of core-shell particles and one visualizing the release of the two actives from the carrier particles. This material is available free of charge via the Internet at http:// pubs.acs.org.

\section{AUTHOR INFORMATION}

\section{Corresponding Author}

m.windbergs@mx.uni-saarland.de; weitz@seas.harvard.edu

Notes

The authors declare no competing financial interest.

\section{ACKNOWLEDGMENTS}

We thank S. Seiffert for his help with confocal microscopy and J. Thiele for support with AutoCAD. J. Vaassen is acknowledged for help with electron microscopy. This work was supported by the German Academic Exchange Service.

\section{REFERENCES}

(1) Allen, T. M.; Cullis, P. R. Science 2004, 303, 1818-1822.

(2) Wong, J. K.; Guenthard, H. F.; Havlir, D. V.; Zhang, Z. Q.; Haase, A. T.; Ignacio, C.; Kwok, S.; Emini, E.; Richman, D. Proc. Natl. Acad. Sci. U.S.A. 1997, 94, 12574-12579.

(3) Jia, J.; Zhu, F.; Ma, X.; Cao, Z.; Li, Y.; Chen, Y. Nat. Rev. Drug Discovery 2009, 8, 111-128.

(4) Kumaravelrajan, R.; Narayanan, N.; Suba, V.; Bhaskar, K. Int. J. Pharm. 2010, 399, 60-70.

(5) McKenna, F. J. Rheumatol. 1998, 25, 21.

(6) Chen, W.; Meng, F.; Cheng, R.; Zhong, Z. J. Controlled Release 2010, 142, 40-46.

(7) Kolishetti, N.; Dhar, S.; Valencia, P. M.; Lind, L. Q.; Karnike, R.; Lippard, S. J.; Langer, R.; Farokhzada, O. C. Proc. Natl. Acad. Sci. U.S.A. 2010, 107, 17939-17944.
(8) Patil, Y.; Sadhukha, T.; Ma, L.; Panyam, J. J. Controlled Release 2009, 136, 21-29.

(9) Jyothi, N. V. N.; Prasanna, P. M.; Sakarkar, S. N. J. Microencapsulation 2010, 27, 187-197.

(10) Witschi, C. Eur. J. Pharm. Biopharm. 1997, 43, 215-242.

(11) Bhymer, C. Pharm. Res. 2003, 20, 337-344.

(12) Okushima, S.; Nisisako, T.; Torii, T.; Higuchi, T. Langmuir 2004, 20, 9905-9908.

(13) Teh, S. Y.; Liu, R.; Hung, L. H.; Lee, A. P. Lab Chip 2008, 8, 198-220.

(14) Dendukuri, D.; Doyle, P. S. Adv. Mater. 2009, 21, 1-16.

(15) Zhang, H.; Tumarkin, E.; Peerani, R.; Nie, Z.; Sullan, R. M. A.; Walker, G. C.; Kumacheva, E. J. Am. Chem. Soc. 2006, 128, 1220512210.

(16) Garti, N. Colloids Surf., A 1997, 123, 233-246.

(17) Zhao, Y.; Shum, H. C.; Chen, H.; Adams, L. L. A.; Gu, Z.; Weitz, D. A. J. Am. Chem. Soc. 2011, 133, 8790-8793.

(18) Khan, A. Y.; Talegaonkar, S.; Iqbal, Z.; Ahmed, F. J.; Khar, R. K. Curr. Drug Delivery 2006, 3, 429-443.

(19) Berndt, I.; Pedersen, J. S.; Richtering, W. J. Am. Chem. Soc. 2005, 127, 9372-9373.

(20) Chu, L. Y.; Xie, R.; Zhu, J. H.; Chen, W. M.; Yamaguchi, T.; Nakao, S. J. Colloid Interface Sci. 2003, 265, 187-196.

(21) Liu, L.; Wang, W.; Ju, X. J.; Xie, R.; Chu, L. Y. Soft Matter 2010, 6, 3759-3763.

(22) P. W. Lee, P. W.; Hsu, S. H.; Tsai, J. S.; Chen, F. R; Huang, P. J.; Ke, C. J.; Liao, Z. X.; Hsiao, C. W.; Lin, H. J.; Sung, H. W. Biomaterials 2010, 31, 2425-2434.

(23) Seiffert, S.; Thiele, J.; Abate, A. R.; Weitz, D. A. J. Am. Chem. Soc. 2010, 132, 6606-6609.

(24) Porter, C. J. H.; Trevaskis, N. L.; Charman, W. N. Nat. Rev. Drug Discovery 2007, 6, 231-248.

(25) Humberstone, A. J.; Charman, W. N. Adv. Drug Delivery Rev. 1997, 25, 103-128.

(26) Yourick, J. J. Int. J. Toxicol. 2010, 29, 123-132.

(27) Utada, A. S.; Lorenceau, E.; Link, D. R.; Kaplan, P. D.; Stone, H. A.; Weitz, D. A. Science 2005, 308, 537-541.

(28) Sun, B. J.; Shum, H. C.; Holtze, C.; Weitz, D. A. ACS Appl. Mater. Interfaces 2010, 2, 3411-3416.

(29) Xu, Q.; Nakajima, M. Appl. Phys. Lett. 2004, 85, 3726-3728.

(30) Guse, C.; Koennings, S.; Maschke, A.; Hacker, M.; Becker, C.; Schreiner, S.; Blunk, T.; Spruss, T.; Goepferich, A. Int. J. Pharm. 2006, 314, 153-160.

(31) Nguyen, C. L.; Eichwald, C.; Nibert, M. L.; Munger, K. J. Virol. 2007, 81, 13533-13543.

(32) Puck, T. T.; Marcus, P. I.; Cieciura, S. J. J. Exp. Med. 1956, 103, $273-283$. 